\title{
Identifying the Pathway to Conservative Pelvic Floor Physiotherapy in a Tertiary Public Hospital in Australia: A Retrospective Audit
}

\author{
Tara Beaumont \\ Women's and Children's Hospital, tara.beaumont@sa.gov.au \\ Kate Goode \\ Women's and Children's Hospital, kate.goode@sa.gov.au
}

Follow this and additional works at: https://nsuworks.nova.edu/ijahsp

Part of the Medicine and Health Sciences Commons

\begin{abstract}
Recommended Citation
Beaumont T, Goode K. Identifying the Pathway to Conservative Pelvic Floor Physiotherapy in a Tertiary Public Hospital in Australia: A Retrospective Audit. The Internet Journal of Allied Health Sciences and Practice. 2017 Jan 01;15(2), Article 3.
\end{abstract}

This Manuscript is brought to you for free and open access by the College of Health Care Sciences at NSUWorks. It has been accepted for inclusion in Internet Journal of Allied Health Sciences and Practice by an authorized editor of NSUWorks. For more information, please contact nsuworks@nova.edu. 


\title{
Identifying the Pathway to Conservative Pelvic Floor Physiotherapy in a Tertiary Public Hospital in Australia: A Retrospective Audit
}

\begin{abstract}
Introduction: Incontinence and/or pelvic organ prolapse symptoms are prevalent in women in Australia and the estimated healthcare cost is considerable. Internationally accepted best practice evidence supports a trial of conservative Physiotherapy treatment with a Physiotherapist trained in the management of pelvic floor dysfunction before surgical options should be considered. Existing referral pathways in the tertiary healthcare system to access conservative Physiotherapy treatment require direct referral from Gynaecology staff. Objective: To identify the pathway to conservative pelvic floor physiotherapy for women referred to a tertiary hospital in Australia with incontinence and/or pelvic organ prolapse symptoms following existing referral pathways. Results: Attendance data for the period January to June 2015 identified 63 women as being primarily referred for incontinence and/or pelvic organ prolapse by their local healthcare provider. Half of these 63 women $(n=31)$ were referred on to Physiotherapy for conservative management; the other half were not. The time between local healthcare provider referral to specialist Gynaecology appointment ranged from 49 days to 351 days (average 169.6 days). Of the 31 women referred to Physiotherapy, the majority $(n=22,70.9 \%)$ were referred on the day of their initial Gynaecology consultation, the 9 remaining women waited between 2 to 126 days for Physiotherapy referral. The shortest pathway from local healthcare provider referral to Physiotherapy consultation was 64 days; the longest pathway was 402 days. Of the 31 women who were not referred to Physiotherapy, 18 (58\%) proceeded directly to surgery. Conclusion: This audit demonstrates that existing referral pathways for women referred with incontinence and/or pelvic organ prolapse create lengthy delays between local healthcare provider referral and access to Physiotherapy services. Only half of those women who would be appropriate for a trial of conservative therapy are being referred to Physiotherapy which is in direct contrast to the widely accepted best practice guidelines for the management of pelvic floor dysfunction mandating conservative therapy as a first line approach for these conditions. The opportunity to implement an advanced scope model of care that promotes more timely access and earlier commencement of conservative Physiotherapy for these women would significantly improve the clinical care pathway for this cohort of patients in the tertiary healthcare setting.
\end{abstract}

\section{Author Bio(s)}

Tara Beaumont, B. App Sc (Physio), PG Cert (Cont PF Rehab), completed her Bachelor of Science (Physiotherapy) in 2001 at the University of South Australia. She has been employed at the Women's and Children's Hospital since 2002. After completing her Post Graduate Certificate (Continence and Pelvic Floor Rehabilitation) at the University of Melbourne in 2006, she was appointed to the Head of Unit, Women's Health Physiotherapy position in 2007. She is currently job sharing this role with Kate Goode after a period of maternity leave.

Kate Goode, B. Physio, APAM, completed her Bachelor of Physiotherapy in 2010 at the University of South Australia. She has been employed at the Women's and Children's Hospital since 2011. She completed her Post Graduate Certificate (Pelvic Floor Rehabilitation) in 2014 and Master of Physiotherapy in 2015 at the University of Melbourne. She has been job sharing the role of Head of Unit, Women's Health Physiotherapy since 2013. 


\title{
IJAHSP \\ The Internet Joưnal of Allied Health Sciences and Practice
}

Dedicated to allied health professional practice and education

Vol. 15 No. 2 ISSN 1540-580X

\section{Identifying the Pathway to Conservative Pelvic Floor Physiotherapy in a Tertiary Public Hospital in Australia: A Retrospective Audit}

\author{
Tara Beaumont, B. App Sc (Physio), PG Cert (Cont PF Rehab) \\ Kate Goode, B. Physio, APAM
}

Women's and Children's Hospital

Australia

\begin{abstract}
Introduction: Incontinence and/or pelvic organ prolapse symptoms are prevalent in women in Australia, and the estimated healthcare cost is considerable. Internationally accepted best practice evidence supports a trial of conservative physiotherapy treatment with a physiotherapist trained in the management of pelvic floor dysfunction before surgical options should be considered. Existing referral pathways in the tertiary healthcare system to access conservative physiotherapy treatment require direct referral from gynaecology staff. Objective: To identify the pathway to conservative pelvic floor physiotherapy for women referred to a tertiary hospital in Australia with incontinence and/or pelvic organ prolapse symptoms following existing referral pathways. Results: Attendance data for the period January to June 2015 identified 63 women as being primarily referred for incontinence and/or pelvic organ prolapse by their local healthcare provider. Half of these 63 women $(n=31)$ were referred on to physiotherapy for conservative management; the other half were not. The time between local healthcare provider referral to specialist gynaecology appointment ranged from 49 days to 351 days (average 169.6 days). Of the 31 women referred to physiotherapy, the majority (n $=22,70.9 \%$ ) were referred on the day of their initial gynaecology consultation; the 9 remaining women waited between 2 to 126 days for physiotherapy referral. The shortest pathway from local healthcare provider referral to physiotherapy consultation was 64 days; the longest pathway was 402 days. Of the 31 women who were not referred to physiotherapy, 18 (58\%) proceeded directly to surgery. Conclusion: This audit demonstrates that existing referral pathways for women referred with incontinence and/or pelvic organ prolapse create lengthy delays between local healthcare provider referral and access to physiotherapy services. Only half of those women who would be appropriate for a trial of conservative therapy are being referred to physiotherapy, which is in direct contrast to the widely accepted best practice guidelines for the management of pelvic floor dysfunction mandating conservative therapy as a first line approach for these conditions. The opportunity to implement an advanced scope model of care that promotes more timely access and earlier commencement of conservative physiotherapy for these women would significantly improve the clinical care pathway for this cohort of patients in the tertiary healthcare setting.
\end{abstract}

\section{INTRODUCTION}

Incontinence (urinary and/or anal) and pelvic organ prolapse symptoms are prevalent in women in Australia, and these conditions can significantly impact a woman's health and wellbeing. Urinary incontinence affects up to $37 \%$ and faecal incontinence affects up to $13 \%$ of Australian women and up to 1 in 2 women who have had children will have some degree of pelvic organ prolapse on vaginal examination. ${ }^{1,2}$ The lifetime risk of surgery for incontinence and pelvic organ prolapse symptoms in women has been shown to be $11.8 \%$ to age 80 , meaning that approximately 1 in 10 women will require surgical intervention for these conditions. ${ }^{3}$ While both incontinence and pelvic organ prolapse represent a significant economic burden on the nation, most literature focuses on the impact of incontinence in particular. The total financial cost of incontinence was estimated to be $\$ 42.9$ billion in 2010 , with an additional $\$ 23.8$ billion if the cost of the burden of disease was also considered. ${ }^{4}$ Internationally accepted best practice evidence strongly supports the role of physiotherapy in the management of pelvic floor dysfunction and, by way of Grade A recommendations using the Oxford Centre of Evidence Based Medicine level of evidence system, mandates the involvement of physiotherapy in the clinical care pathway for these conditions: 5 
- Supervised pelvic floor muscle training should be offered as first line treatment for urinary incontinence (Grade A recommendation)

- Conservative physiotherapy management can reduce pelvic organ prolapse symptoms and reduce patient-reported symptom severity (Grade A recommendation)

The existing referral pathway at the Women's and Children's Hospital (WCH; Adelaide, South Australia) for women with incontinence and/or pelvic organ prolapse symptoms to access conservative physiotherapy management requires referral from their local medical caregiver (often a general practitioner (GP)) to the specialist gynaecology outpatient clinics. These referrals are triaged by senior medical staff in the Gynaecology Department as low priority "P3" for an initial consultation appointment (despite being a high priority for physiotherapy input), and thus, may wait in excess of 6 months for an appointment with the Gynaecology Team, at which point the patient must choose to "opt in" to the service. Once they have been seen in the Gynaecology Clinic, if appropriate, they are then referred to Women's Health Physiotherapy to a trial period of conservative treatment (usually 4-6 months of therapy, which generally equates to $5-6$ occasions of service). At this point, surgical management may be considered in such cases where it appears that conservative management has failed, and symptom severity and bother have not improved sufficiently from the patient's perspective.

The pilot Gynaecology Physiotherapy Assessment Service (GPAS) was implemented in October 2015, an initiative designed to improve the clinical care pathway for women referred to the Gynaecology Department at the WCH with symptoms of incontinence (urinary and/or anal) and pelvic organ prolapse, or a combination of these conditions. The GPAS model of care aims to facilitate more timely access to appropriate care in the tertiary healthcare system, while ensuring better utilisation of medical staff time and resources. By implementing a physiotherapy-led assessment service, women referred with mild or moderate pelvic floor dysfunction symptoms can be diverted from the existing waitlist to see a gynaecologist and instead be seen in a much timelier manner by a physiotherapist trained in the assessment and management of pelvic floor dysfunction. The GPAS model allows those women who are responding favourably to conservative management to be removed from the gynaecology waitlist, while also allowing those who require specialist medical review and/or surgical opinion to keep their place on the waitlist to see the gynaecologist. As a result, this pathway helps to ensure that gynaecology clinics are appropriately booked with patients who will require the Gynaecology Team's specialist medical knowledge and/or surgical opinion, thereby improving the utilisation of specialist medical staff time and resources.

The GPAS clinic is the first of its kind to run in the public sector in South Australia and is the first advanced scope role for physiotherapy in the Women's and Babies' Division at the WCH. An advanced scope role is defined as "a role that is within the currently recognised scope of practice for a profession, but that through custom and practice, has been performed by other professions. The advanced role may require additional training as well as significant professional experience and competency development." 6 The GPAS clinic model closely resembles similar advanced scope clinics operating in other Australian states. Informal correspondence with the lead physiotherapists for some of these clinics suggest that the roles have resulted in more timely access to conservative management, reduced symptom severity, reduction in patients on the gynaecology waitlist, improved patient flow through the health services, and high patient satisfaction since their inception. 
Figure 1: Existing Pathway to Conservative Management

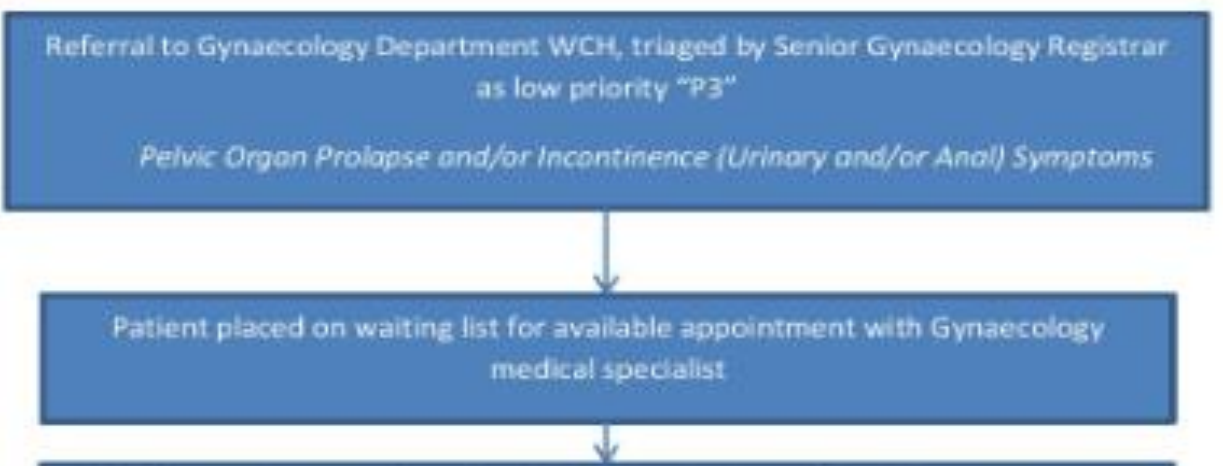

Available appointment times identifled $\rightarrow$ patients on P3 wat list are contacted

by letter and must "opt in" for review via return correspondence.

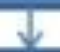

Patient attends WCH Gynaecolosy clinic for medical specialist axsessment and further investigations as indicated

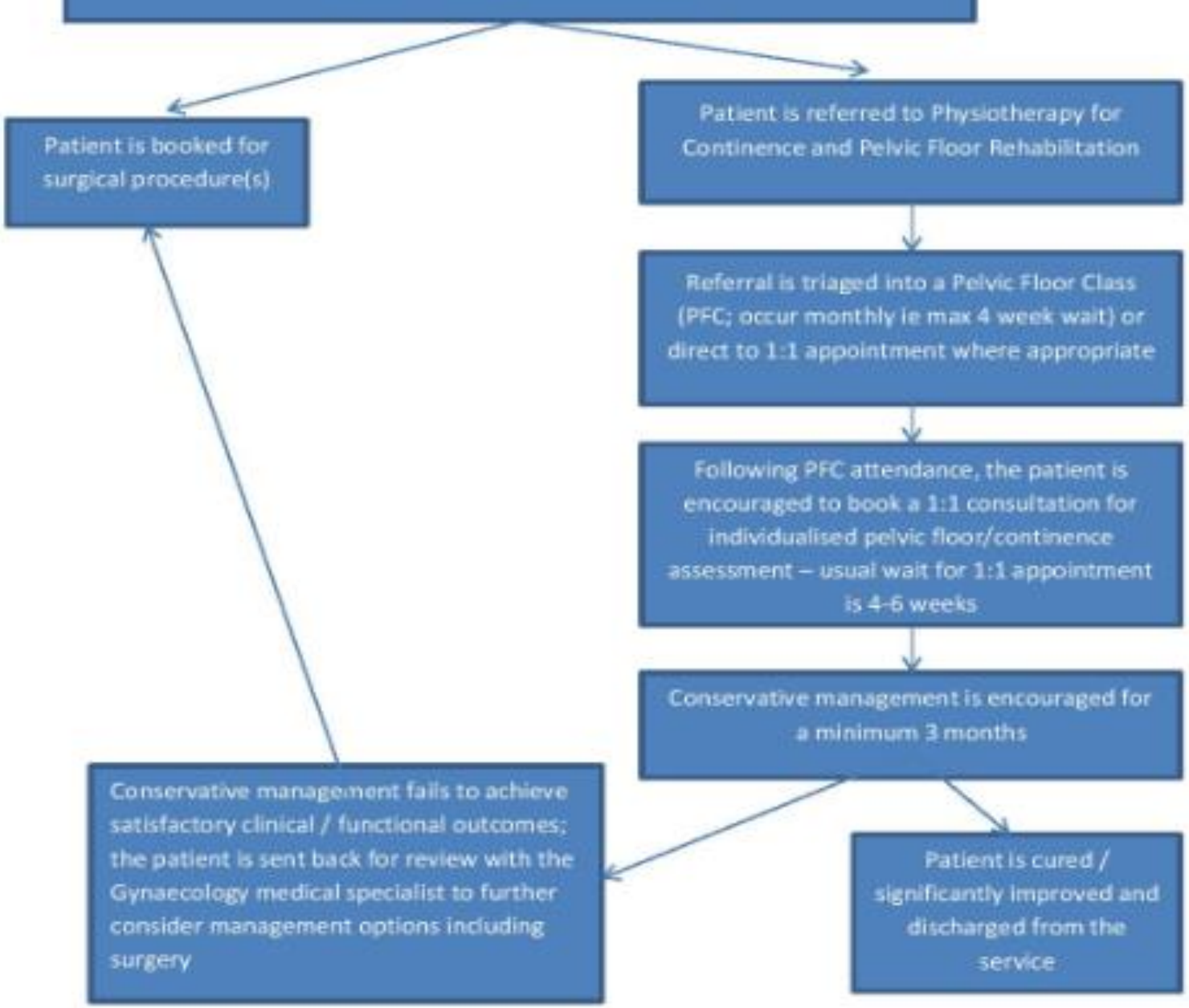




\section{Method}

A retrospective audit was conducted to determine the pathway for a cohort of women who met GPAS inclusion criteria but who followed the existing referral pathway prior to the establishment of the GPAS pilot. GPAS inclusion criteria were defined as incontinence (urinary and/or anal symptoms), pelvic organ prolapse (grade I - III), or a mixed presentation of incontinence and/or pelvic organ prolapse symptoms. Ethics approval for this audit was granted by the WCH Human Research Ethics Committee in July 2015 (Audit 797A).

A review of the hospital's Clinical Information Systems (CIS) data showed 498 occasions of service provided by gynaecology staff to patients triaged as "P3" between Jan $1^{\text {st }}$ and June $30^{\text {th }} 2015$. A list of these occasions of service was generated by CIS personnel, including patient name, date of birth, hospital identifier number, date of GP/medical caregiver referral, and date of Gynaecology Clinic presentation. Each of the medical records for these identified patients were recalled and reviewed, with key information recorded on an Excel spreadsheet.

- Would the patient have been appropriate for GPAS (based on GP/medical caregiver referral letter detail) - yes or no?

- If no, was the patient referred to physiotherapy for a relevant secondary symptom (that was not flagged on the original GP/medical caregiver referral letter)?

$\circ \quad$ If yes, was the patient referred to physiotherapy - yes or no?

- If yes, when did this referral to physiotherapy occur - date?

- If yes, did the patient attend their physiotherapy appointment - yes or no?

- If yes, what has been the patient journey since referral to physiotherapy - free text e.g., attending for ongoing physiotherapy treatment, failed to attend subsequent appointments, pursued surgical intervention?

- If no, what has been the patient journey since presentation to the Gynaecology Outpatient Clinic free text e.g., proceeded to surgery?

The time difference (days) between referral from GP/medical caregiver to the Gynaecology Outpatient Clinic appointment for those patients who would have met GPAS inclusion criteria was calculated. For those patients who were then subsequently referred to physiotherapy, the time difference (days) between gynaecology appointment and referral to physiotherapy, as well as the time difference (days) between date of referral to physiotherapy and initial consultation with the physiotherapy service, were also calculated.

\section{Results}

Data obtained via the WCH Clinical Information Service indicated 498 occasions of service to women triaged as "P3" were provided through the outpatient Gynaecology Clinics for the period January 1 to June 30 2015. On further review, this represents 377 individual patients seen during this time period. Women who were triaged as "P3" by senior gynaecology staff and who attended the Gynaecology Clinic during this time were referred for a variety of reasons by their medical caregiver (primarily a local GP), including infertility, contraception, dysfunctional and/or heavy uterine bleeding, incontinence and pelvic organ prolapse, or a combination of these reasons. When considering the reason for the referral against the inclusion criteria for GPAS, 63 women would have met the criteria for the fast-tracked GPAS pathway (16.7\%). It should be noted that an additional 6 women were referred to the Gynaecology Clinic by WCH physiotherapy staff, and these women were excluded from further analysis as they were currently accessing physiotherapy care at the time of referral (and beyond). Of the 308 patients who would not have met the criteria for GPAS (based on the referring information i.e., no mention of continence issues and/or pelvic floor dysfunction), 18 of these were subsequently referred to physiotherapy for continence and/or pelvic floor muscle rehabilitation after attending their outpatient gynaecology appointment.

The pathway for the cohort of women who would have been eligible for GPAS was further reviewed and analysed. Of the 63 women who would have been eligible for GPAS, 31 of these were referred to physiotherapy, and 31 were not referred to physiotherapy one patient was already receiving physiotherapy input for the reason outlined on her referral to gynaecology (but had self-referred appropriately to physiotherapy for input prior to GP referral) and her pathway was not considered further. In essence, only half of the women who were appropriate for physiotherapy intervention were referred to physiotherapy during this 6-month period.

Of the 31 women who were referred to physiotherapy after attending their Gynaecology Clinic appointment, 13 were diagnosed with incontinence (predominantly urinary) symptoms, 10 with pelvic organ prolapse, and 8 with a combination of both incontinence and pelvic organ prolapse symptoms. The time between GP/medical caregiver referral to the Gynaecology Clinic appointment ranged

(c) The Internet Journal of Allied Health Sciences and Practice, 2017 
from 49 days to 351 days, with an average 169.6 days. In 22 cases, the women were referred to physiotherapy on the same day as the first Gynaecology Clinic appointment; the remaining 9 women waited a further 2 to 126 days (average 78.1 days) before a referral was made to physiotherapy. Patients waited on average 32.8 days (range 7 to 66 days) between referral to physiotherapy and attendance at a physiotherapy appointment. The quickest individual pathway from GP referral to physiotherapy appointment was 64 days; the longest pathway from GP to physiotherapy appointment was 402 days.

Once a referral had been made to physiotherapy, 8 women declined or did not attend their initial physiotherapy appointment (25.8\%). Of those who attended physiotherapy, the majority $(20 / 31,64.5 \%)$ attended a group pelvic floor class. There were 3 women who were triaged as not appropriate for the group session and were seen individually as the first contact with the physiotherapy department. After attending a pelvic floor class, individual 1:1 appointments were offered to each patient; this was taken up by 11 women, many of whom went on to attend 2 to 5 individual therapy sessions before being discharged or proceeding with gynaecological surgery. Further in-depth analysis of patient outcome is limited by the time frame of the study, as many women were still on current physiotherapy treatment as this report was being completed.

Of the 31 women who were suitable for GPAS inclusion but who were not referred for physiotherapy, 18 of those (58\%) went straight to surgical management, with another patient considering surgical management at the time of audit.

\section{Discussion}

This audit highlights several shortcomings with the existing pathway into physiotherapy for women with incontinence and/or pelvic organ prolapse symptoms. Women with symptoms that could be managed by an experienced physiotherapist trained in the management of pelvic floor dysfunction are triaged as a lower priority by the Gynaecology Department and are waiting on average 5-6 months - some close to 12 months - before preliminary consultation through the Gynaecology Clinic occurs and referral to physiotherapy is made. In a community setting, the physiotherapist essentially acts as the first point practitioner as referral from a medical caregiver is not required to access private physiotherapy care. The new GPAS model aims to mimic this first point practitioner role but also with advanced scope of practice to screen and identify any potential yellow/red flags or conditions requiring specific specialised medical and/or gynaecological review. In the advanced scope role, the physiotherapist in GPAS is able to complete standard urinalysis (via urine dipstick) and screen for pathological changes in urine, assess post void residual volumes, refer the patient for urodynamic assessment (following discussion and liaison with gynaecology staff), recommend relevant treatments via the patient's GP (e.g., topical oestrogen for vaginal atrophy), and screen for any red flags that may indicate potentially sinister pathology (for example, abnormal vaginal bleeding or unexplained weight loss). The GPAS pathway allows a more streamlined and efficient medical service for these women and has the potential to uncover conditions requiring specific specialist medical management in a more timely way than the existing pre-GPAS pathway.

This audit indicated that only $50 \%$ of the women who should have been referred to physiotherapy (based on the information on the referral letter) for a trial of conservative management are being referred for physiotherapy input at the WCH. Additionally, even when women are being referred for physiotherapy input, some are waiting in excess of a year to access physiotherapy care. Internationally accepted Grade A recommendations mandate physiotherapy input as first line therapy in the clinical care pathway of women with urinary incontinence and pelvic organ prolapse symptoms. ${ }^{5}$ High quality evidence supporting the effectiveness of pelvic floor muscle training (PFMT) in curing and/or improving patients with urinary incontinence exists, as outlined in a recent systematic review by Dumoulin et al that reviewed 18 randomised or quasi randomised trials with a total of 1281 female participants. ${ }^{7}$ Similarly, Hagen et al have recently completed the largest, most rigorous study of its kind which looked at 447 women with stage I-III pelvic organ prolapse in a randomised controlled trial, showing that PFMT significantly reduced pelvic organ prolapse symptoms in the intervention group. ${ }^{8}$ Those women who do go on to require surgery are more likely to have favourable outcomes because of the education and training they receive during their physiotherapy treatment. ${ }^{9}$ In keeping with a study by Neumann et al, it is expected that a significant proportion of the women who are assessed by the physiotherapist in the new GPAS model and referred directly to the Women's Health Physiotherapy Department for input will be cured or have a significant reduction in their clinical symptoms such that they may be removed from the Gynaecology Clinic waiting list and/or avoid the need for surgery. ${ }^{10}$

A proportion of the 31 women (8/31) who were not referred to physiotherapy (but who met GPAS inclusion criteria) were reported to have received physiotherapy management previously with little to no success. However, it is not clear where this physiotherapy management was provided, what it consisted of, or if the physiotherapist was specifically trained or experienced in the management of these conditions. The opportunity to provide physiotherapy input and training - even when surgery is inevitable or desired - has been shown to improve post-surgical outcomes for these patients. ${ }^{9}$ For women who wish to avoid surgery and who have tried conservative management previously, a repeat course of conservative therapy should still be strongly considered as there are often many contributing factors to conservative management failure, and these factors can often change with time and perspective. It has been shown that supervised pelvic floor muscle training in a tertiary healthcare setting with a trained physiotherapist can have positive

(C) The Internet Journal of Allied Health Sciences and Practice, 2017 
clinical outcomes for women with stress incontinence symptoms, even when prior attempts at conservative management in the community have seemingly failed.11

The attendance rate following physiotherapy referral is pleasing $(74.2 \%$ when combining the pelvic floor class attendees with those who attended an individual appointment as first contact with the physiotherapy department), but it is disappointing that only $55 \%$ of women who attended the pelvic floor class appointment continued through to individual 1:1 appointments. The pelvic floor class group education session is important as it allows a baseline level of knowledge to be imparted to all women regarding pelvic floor muscle anatomy and function, as well as normal bladder and bowel function, while also allowing theoretical teaching of correct pelvic floor muscle contraction (and relaxation). Participants leave the session with "homework" e.g., completion of a fluid balance chart or accident diary, which provides valuable clinical information during the individual consultation appointment. Attendance at the group education session allows the physiotherapist to assume a baseline level of knowledge and understanding, thereby streamlining the individual appointment and facilitating a quicker tailored plan of intervention with a well-informed patient. The provision of group education about pelvic floor muscle anatomy and function, as well as bladder and bowel function, ahead of individual consultation is supported by Grimshaw who reported that women found this specific type of group session helpful, enjoyable, and informative. ${ }^{12}$ Many reported an improvement in their symptoms prior to individual physiotherapy appointment after group education attendance and were noticeably more relaxed about digital vaginal examination in this consultation than those who had not attended the group session.

The pelvic floor muscle contraction technique is assessed by digital vaginal examination (with consent) in the individual physiotherapy appointment, and this is a key aspect of care. Evidence suggests that even with comprehensive written and verbal instruction, many women still perform their pelvic floor muscle exercises incorrectly. ${ }^{13}$ It is concerning that in this audit, nearly half of the women who attended the group education session did not go on to have this individual assessment. As such, the clinical effectiveness of conservative treatment is likely to be compromised for these women. In-depth analysis with respect to the reason why women did not pursue individual pelvic floor assessment after group session attendance fell outside the scope of this audit but may be a valuable piece of work to complete. It may be hypothesised that the time lag between initial referral from medical caregiver to physiotherapy was such that patient motivation for conservative treatment was affected and attrition away from conservative management was higher than expected. It can be noted that at least one patient (of the 9 who did not attend individual assessment appointment) was booked for surgery in the weeks following group education session attendance. The time constraints of the audit may also have contributed to this finding (i.e., individual assessment may be planned but not yet attended at the time of the report). One of the clear benefits of the GPAS pathway is that digital vaginal examination and pelvic floor assessment at the time of GPAS appointment allows for more timely correction/modification (if required) of pelvic floor muscle contraction technique, and this can have a significant impact on the presence and severity of symptoms for these women. The GPAS model also offers the opportunity to actively promote the benefits of conservative physiotherapy management for these women early in their clinical care pathway and facilitate access to conservative treatment in a more timely manner, which may impact positively on the attrition rate seen in this audit.

\section{Conclusion}

This retrospective audit highlights lengthy wait times to see a gynaecologist and physiotherapist for patients triaged as a low priority "P3" with incontinence and/or pelvic organ prolapse symptoms with the existing referral pathway in a tertiary healthcare setting. As such, the opportunity for timely physiotherapy intervention and therefore the ability to positively influence clinical outcomes is compromised. In addition, only half of patients who are appropriate for physiotherapy as first line treatment for incontinence and/or pelvic floor dysfunction are currently referred for conservative management at this centre. An advanced scope physiotherapy role, acting as a first point practitioner for these women, is likely to provide a more efficient pathway for access to conservative management. Patients responding positively to conservative management could then be removed from the gynaecology waiting list, meaning that those who remain on the wait list to see a gynaecologist are more appropriate and indeed do need specialist medical knowledge and intervention.

\section{References}

1. Australian Incontinence Data Analysis and Development. Australian Institute of Health and Welfare (AlHW), 2006. Canberra: AlHW. Available at: http://www.aihw.gov.au/WorkArea/DownloadAsset.aspx?id=6442455813. Accessed July 21, 2015.

2. Barber M, Maher C. Epidemiology and outcome assessment of pelvic organ prolapse. Int Urogynecol J. 2013 Nov; 24(11):1783-90. [PMID: 24142054]

3. Fialkow $M$, Newton $K$, Lentz $G$, Weiss $N$. Lifetime risk of surgical management for pelvic organ prolapse or urinary incontinence. Int Urogynaecol J Pelvic Floor Dysfunct. 2008 March;19(3):437-40. [PMID: 17896064]

4. The Economic Impact of Incontinence in Australia. Continence Foundation of Australia, 2011. Available at: http://www.continence.org.au/data/files/Access_economics_report/dae_incontinence_report_19_april_2011.pdf. Accessed July 21, 2015.

(C) The Internet Journal of Allied Health Sciences and Practice, 2017 
5. Moore K, Dumoulin C, Bradley C, Burgio K, Chambers T, Hagen S, Hunter K, Imamura M, Thakar R, Williams K. Committee 12: Adult Conservative Management. In Abrams P, Cardozo L, Khoury S, Wein A (Eds): Incontinence: 5th International Consultation on Incontinence. Plymbridge United Kingdom: Health Publication Ltd; 2013:1101-227.

6. Australian Physiotherapy Association Position Statement: Scope of Practice. Australian Physiotherapy Association, 2009. Available at: https://www.physiotherapy.asn.au/DocumentsFolder/Advocacy_Position_Scope_of_Practice_2009.pdf. Accessed March 24, 2016.

7. Dumoulin C, Hay-Smith J, Habée-Séguin GM, Mercier J. Pelvic floor muscle training versus no treatment or inactive control treatments, for urinary incontinence in women: a short version Cochrane systematic review with meta-analysis. Neurourol Urodynam. 2015 Apr;34(4):300-8. [PMID: 25408383]

8. Hagen S, Stark D, Glazener C, Dickson S, Barry S, Elders A, Frawley H, Galea MP, Logan J, McDonald A, McPherson G, Moore KH, Norrie J, Walker A, Wilson D, POPPY Trial Collaborators. Individualised pelvic floor muscle training in women with pelvic organ prolapse (POPPY): a multicentre randomised controlled trial. Lancet. 2014 Mar;383(9919):796-806. [PMID: 24290404]

9. Jarvis $S$, Hallam T, Lujic S, Abbott J, Vancaillie T. Peri-operative physiotherapy improves outcomes for women undergoing incontinence and or prolapse surgery: results of a randomised controlled trial. Aust N Z J Obstet Gynaecol. 2005 Aug;45(4):300-3. [PMID: 16029296]

10. Neumann P, Grimmer K, Grant R, Gill V. Physiotherapy for female urinary stress incontinence: a multi-centre observational trial. Aust N Z J Obstet Gynaecol. 2005 June;45(3):226-32. [PMID: 15904449]

11. Slack $A$, Hill $A$, Jackson $S$. Is there a role for a specialist physiotherapist in the multi-disciplinary management of women with stress incontinence referred from primary care to a specialist continence clinic? J Obstet Gynaecol. 2008 May:28(4):410-2. [PMID: 18604676]

12. Grimshaw R. An audit of physiotherapy classes for women with pelvic floor dysfunction. J Assoc Charter Physiother Women's Health. 2005 Spring;96:62-4.

13. Benvenuti $F$, Caputo GM, Bandinelli S, Mayer F, Biagini C, Sommavilla A. Reeducative treatment of female genuine stress incontinence. Am J Phys Med. 1987 Aug;66(4):155-68. [PMID: 3674220] 